\title{
Microwave - Assisted Synthesis of a Library of Pyrrolo[1,2-c]quinazolines
}

\author{
EMILIAN GEORGESCU1, FLOREA DUMITRASCU², FLORENTINA GEORGESCU ${ }^{3}$, CONSTANTIN DRAGHICI², \\ DENISA DUMITRESCU4* \\ ${ }^{1}$ Chimcomplex Rm. Valcea, Research Center, 1 Uzinei Str., 240050, Ramnicu Valcea, Romania \\ ${ }^{2}$ Center of Organic Chemistry C. D. Nenitzescu, Romanian Academy, 202B Splaiul Independentei, 060023, Bucharest, \\ Romania \\ ${ }^{3}$ Enpro Soctech Com srl, 31 Elefterie Str., 050524, Bucharest, Romania \\ ${ }^{4}$ Ovidius University of Constanta, Faculty of Pharmacy, 6 Capitan Aviator Al. Serbanescu Str., 900470, Constanta, Romania
}

\begin{abstract}
A library of new pyrrolo[1,2-c]quinazoline derivatives was obtained by the one-pot, three-component microwave-assisted synthesis, starting from quinazolines, 2-bromoacetyl derivatives and electron-deficient alkynes in 1,2-epoxybutane via 1,3-dipolar cycloaddition of quinazolinium N-ylides. This synthetic pathway offers a simple and rapid access to a large range of pyrrolo[1,2-c]quinazoline derivatives.
\end{abstract} Keywords: pyrrolo[1,2-c] quinazolines, microwave-assisted synthesis, one-pot three components reactions,
1,3-dipolar cycloadditions

Pyrrolo[1,2-c]quinazoline, an interesting [5,6]-fused ring system with a bridgehead nitrogen atom, is the core of several bioactive pyrrolo[1,2-c]quinazoline derivatives effective in the treatment of cardiovascular disorders and as antiasthmatic agents [1]. Some pyrrolo[1,2-c]quinazolin5 -one compounds are valuable building blocks in the synthesis of potentially bioactive heterocycles [2].

Synthetic strategies toward pyrrolo[1,2-c]quinazoline derivatives have been reviewed in recent years [3].The main synthetic routes toward pyrrolo[1,2-a]quinoline derivatives start from substituted 2-arylpyrroles [4-8], and from quinazolines either by catalyzed [ $3+2$ ] annulation of substituted 4-trifluoromethyl-1,2-dihydroquinazolin-2-ones with Morita-Baylis-Hillman carbonates [9] and ethyl 2,3butadienoate [10], or by the intramolecular cyclization reaction of 3-vinyl-3,4-dihydroquinazolin-2-one in the presence of tetrabutylammonium fluoride [11]. Many pyrrolol[1,2-c]quinazoline derivatives were synthesized by 1,3-dipolar cycloaddition reactions of the $\mathrm{Fe}^{3+}$-stabilized azomethine ylides with allenoates, alkynoates and $\alpha$-amino acid methyl esters [3,12] or by 1,3-dipolar cycloaddition reactions of quinazolinium $N$-ylides with various alkyne dipolarophiles [13-19]. The classical synthetic procedure for the 1,3-dipolar cycloaddition reactions of heterocyclic $N$ ylides involvesthe following steps: the preparation of $\mathrm{N}$ heterocyclic salts by the quaternization of $N$-heterocycle with 2-bromoacetophenones, the in situ generation of the heterocyclic $\mathrm{N}$-ylides from the corresponding heterocyclicsalts under the action of bases and the1,3-dipolar cycloaddition reactions of heterocyclic $\mathrm{N}$-ylides with acetylenic dipolarophiles [20,21]. The known instability of the most of quinazolinium salts due to the electron deficiency in the position 4 of the quinazoline nucleus $[13,14,22-24]$, which is the main disadvantage of this synthetic procedure, has been overcome by developing a multicomponent methodology for the synthesis of pyrrolo[1,2-c]quinazolines. This one-pot, three component syntheticprocedure starts from various substituted quinazolines, 2-bromo-acetophenones and electrondeficient alkynes in the presence of an epoxide which plays both the role of the acid scavenger and reaction solvent [13-19]. However, this synthetic pathway requires a long reaction time, which is an important limitation for synthesizing a large library of pyrrolo[1,2-c]quinazolines. Therefore, an efficient, rapid, and clean synthetic procedure toward libraries of such compounds is still an important issue.

Microwave irradiation offers a facile and versatile method for the synthesis of fused heterocyclic compounds. This method substantially decreases the reaction time, is less solvent and energy consumer and improves the yields [25-32].

As a continuation of our interest in this field [13$19,24,30]$,we report here a one-pot, three components microwave-assisted synthesis of a library of pyrrolo[1,2c] quinazoline which has the advantages of considerable shorter reaction time, reduced solvent spending and good yields.

\section{Experimental part \\ General information}

Microwave-assisted synthesis were carried out using a Biotage Initiator 2.0 EXP - ED instrument (Biotage AB, USA). Melting points were determined on a Boëtius hot plate microscope. The IR spectra were recorded on a Nicolet Impact 410 spectrometer, in $\mathrm{KBr}$ pellets. The NMR spectra were recorded on a Varian Gemini $300 \mathrm{BB}$ instrument, operating at $300 \mathrm{MHz}$ for ${ }^{1} \mathrm{H}-\mathrm{NMR}$ and $75 \mathrm{MHz}$ for ${ }^{13} \mathrm{C}-\mathrm{NMR}$. Supplementary evidence was given by HETCOR and COSY experiments.The elemental analysis was carried out on a COSTECH Instruments EAS32 apparatus.Satisfactory microanalyses for all new compounds were obtained.

The quinazoline and halogeno-substituted quinazolines were obtained starting from 2-nitrobenzaldehyde by Riedel synthesis [22] . 2-Bromo-1'-acetonaphthone was prepared from 1-acetylnaphthalene by the bromination with bromine in diethyl ether. 1-Phenyl-2-propyn-1-one was obtained by the oxidation of 1-phenyl-2-propyn-1-ol with $\mathrm{CrO}_{3}$ in acidic media. The other quaternizing agents, electron-deficient alkynes and 1,2-epoxybutane were commercially products and used without supplementary purifications.

General procedure for microwave-assisted syntheses of pyrrolo[ 1,2-c[quinazolines (4-26)

A mixture of quinazoline derivative1 (2 mmole), 2bromoacetophenone $\mathbf{2}$ ( 2 mmole) and activated alkyne $\mathbf{3}$ 
(2.2 mmole) in $18 \mathrm{~mL}$ 1,2-epoxybutane was placed into a sealed microwave reactor at $120^{\circ} \mathrm{C}$ for $45 \mathrm{~min}$. The reaction mixture was cooled to room temperature, partly of the solvent was removed in vacuum, $5 \mathrm{~mL}$ of $\mathrm{MeOH}$ was added under a gentle stirring and the reaction mixture was left overnight at $5-10{ }^{\circ} \mathrm{C}$. The solid formed was filtered-off, washed on the filter with a mixture of diethyl ether- $\mathrm{MeOH}$ 2:1 and crystallized from $\mathrm{CHCl}_{3}$ or $\mathrm{CHCl}_{3}$ /Et O.The melting points and yields for all synthesized pyrrolo[1,2c]quinazolines are shown in Table 2 . The spectral data and elemental analysis are given below.

Ethyl 3-(3-trifluoromethylbenzoyl)pyrrolo[1,2c]quinazoline-1-carboxylate (4). Pale yellow crystals (from $\mathrm{CHCl}_{3} / \mathrm{Et}_{2}$ ). ). . ${ }^{\mathrm{H}} \mathrm{H}-\mathrm{NMR}\left(300 \mathrm{MHz}, \mathrm{CDCl}_{3}, \delta \mathrm{ppm}\right.$ ): $1.41(\mathrm{t}, 3 \mathrm{H}, J=7.1 \mathrm{~Hz}, \mathrm{Me}), 4.42\left(\mathrm{q}, 2 \mathrm{H}, J=7.1^{3^{\prime}} \mathrm{Hz}, \mathrm{CH}_{2}\right)$ 7.68- $7.74\left(\mathrm{~m}, 2 \mathrm{H}, \mathrm{H}-8, \mathrm{H}-5^{\prime}\right), 7.77-7.83(\mathrm{~m}, 1 \mathrm{H}, \mathrm{H}-9), 7.81$ $(\mathrm{s}, 1 \mathrm{H}, \mathrm{H}-2), 7.88-7.92\left(\mathrm{~m}, 1 \mathrm{H}, \mathrm{H}-4^{\prime}\right), 8.03(\mathrm{dd}, 1 \mathrm{H}, \mathrm{J}=8.1$, $1.3 \mathrm{~Hz}, \mathrm{H}-7), 8.06-8.10\left(\mathrm{~m}, 1 \mathrm{H}, \mathrm{H}-6^{\prime}\right), 8.15-8.17(\mathrm{~m}, 1 \mathrm{H}, \mathrm{H}-$ $\left.2^{\prime}\right), 9.75(\mathrm{dd}, 1 \mathrm{H}, J=8.3,1.4 \mathrm{~Hz}, \mathrm{H}-10), 10.34(\mathrm{~s}, 1 \mathrm{H}, \mathrm{H}-5)$. ${ }^{13} \mathrm{C}-\mathrm{NMR}\left(75 \mathrm{MHz}, \mathrm{CDCl}{ }_{3}, \delta \mathrm{ppm}\right): 14.3(\mathrm{Me}), 61.0\left(\mathrm{CH}_{2}\right)$, $111.1,119.8,122.9,124.3\left(\mathrm{q}, J=273.2 \mathrm{~Hz}, \mathrm{CF}_{3}\right), 126.0\left(\mathrm{q}^{2}\right.$ $\left.J=3.6 \mathrm{~Hz}, \mathrm{C}-2^{\prime}\right), 127.8(\mathrm{CH}), 128.2(\mathrm{CH}), 128.4(\mathrm{CH}), 128.5$ (CH), $128.6\left(\mathrm{q}, J=3.5 \mathrm{~Hz}, \mathrm{C}-4^{\prime}\right), 130.4(\mathrm{CH}), 131.3(\mathrm{CH})$, $131.3\left(\mathrm{q}, J=32.7 \mathrm{~Hz}, \mathrm{C}-3^{\prime}\right), 131.4(\mathrm{CH}), 132.2(\mathrm{CH}), 137.1$, 138.1 (CH), 139.6, 141.3, 163.5 (COO), 183.9 (CO).IR ( $\mathrm{KBr}$, $\left.\mathrm{cm}^{-1}\right)$ : 3017, 2983, 1718, 1631, 1537, 1453, 1359, 1327, $1229,1192,1156,1129,1097,1072$. Anal. Calcd. for $\mathrm{C}_{2} \mathrm{H}_{15} \mathrm{~F}_{\mathrm{N}} \mathrm{O}$ (412.36): C,64.08; $\mathrm{H}, 3.67 ; \mathrm{N}, 6.79$. Found: C,64.22; $\mathrm{H}, 3.81 ; \mathrm{N}, 6.61$.

1-Benzoyl-3-(3-trifluoromethylbenzoyl)pyrrolo [1,2c] quinazoline (5). Yellow crystals (from $\mathrm{CHCl}_{3} / \mathrm{Et}_{2} \mathrm{O}$ ). ${ }^{1} \mathrm{H}$ NMR ( $300 \mathrm{MHz}, \mathrm{CDCl}_{2} \delta$ ppm): $7.46(\mathrm{~s}, 1 \mathrm{H}, \mathrm{H}-2), 7.49-7.54$ ( $\mathrm{m}, 2 \mathrm{H}$, meta-Ph), 7.59-7.67 ( $\left.\mathrm{m}, 3 \mathrm{H}, \mathrm{H}-8, \mathrm{H}-5^{\prime}, \mathrm{Ph}\right), 7.76-$ $7.81(\mathrm{~m}, 1 \mathrm{H}, \mathrm{H}-9), 7.82-7.85\left(\mathrm{~m}, 1 \mathrm{H}, \mathrm{H}-\mathrm{4}^{\prime}\right), 7.91-7.94(\mathrm{~m}$, $2 \mathrm{H}, \mathrm{Ph}), 8.02-8.06\left(\mathrm{~m}, 2 \mathrm{H}, \mathrm{H}-7, \mathrm{H}-6^{\prime}\right), 8.15-8.17(\mathrm{~m}, 1 \mathrm{H}, \mathrm{H}-$ $\left.2^{\prime}\right), 8.98(\mathrm{dd}, 1 \mathrm{H}, J=8.3,1.4 \mathrm{~Hz}, \mathrm{H}-10), 10.38(\mathrm{~s}, 1 \mathrm{H}, \mathrm{H}-5)$. ${ }^{13} \mathrm{C}-\mathrm{NMR}(75 \mathrm{MHz}, \mathrm{CDCl}, \delta \mathrm{ppm}): 118.1,119.8,122.6,124.3$ $\left(\mathrm{q}, J=277.4 \mathrm{~Hz}, \mathrm{CF}_{3}\right), 126.0\left(\mathrm{q}, J=3.8 \mathrm{~Hz}, \mathrm{C}-2^{\prime}\right), 126.5(\mathrm{CH})$, $128.5(\mathrm{CH}), 128.6(\mathrm{CH}), 128.7(\mathrm{CH}), 128.8(\mathrm{q}, J=3.7 \mathrm{~Hz}, \mathrm{C}-$ $\left.4^{\prime}\right), 129.3(\mathrm{CH}), 129.8(\mathrm{CH}), 130.9(\mathrm{CH}), 131.3(\mathrm{q}, J=32.1$ $\left.\mathrm{Hz}, \mathrm{C}-3^{\prime}\right), 131.7(\mathrm{CH}), 132.2(\mathrm{CH}), 133.1(\mathrm{CH}), 137.0,138.1$ (C-5), 138.8, 139.4, 141.2, 183.9 (CO), 191.4 (CO).IR (KBr, $\left.\mathrm{Cm}^{-1}\right): 3107,1643,1612,1581,1509,1442,1397$, 1331,1259, 1198, 1117, 1065. Anal. Calcd. for $\mathrm{C}_{2} \mathrm{H}_{1} \mathrm{~F}_{3} \mathrm{~N}_{2} \mathrm{O}$ (444.40): $C, 70.27 ; \mathrm{H}, 3.40 ; \mathrm{N}, 6.30$. Found: $\mathrm{C}, 70.43 ; \mathrm{H}, 3.51$; $\mathrm{N}, 6.15$.

Ethyl 3-(3,4-dimethoxybenzoyl)pyrrolo[ 1,2c]quinazoline-1-carboxylate (6). Pale yellow crystals (from CHCl,/Et O). ${ }^{1} \mathrm{H}-\mathrm{NMR}(300 \mathrm{MHz}, \mathrm{CDCl}, \delta \mathrm{ppm}): 1.43$ $(\mathrm{t}, 3 \mathrm{H}, J=7.1 \mathrm{~Hz}, \mathrm{Me}), 3.98,4.01(2 \mathrm{~s}, 6 \mathrm{H}, 2 \mathrm{MeO}), 4.43(\mathrm{q}$, $\left.2 \mathrm{H}, J=7.1 \mathrm{~Hz}, \mathrm{CH}_{2}\right), 7.01\left(\mathrm{~d}, 1 \mathrm{H}, J=8.2, \mathrm{H}-5^{\prime}\right), 7.51(\mathrm{~d}, 1 \mathrm{H}$, $\left.J=1.7, H-2^{\prime}\right), 7.58^{2}\left(\mathrm{dd}, 1 \mathrm{H}, J=8.2,1.7, \mathrm{H}-6^{\prime}\right), 7.67-7.71(\mathrm{~m}$, $1 \mathrm{H}, \mathrm{H}-9), 7.75-7.80(\mathrm{~m}, 1 \mathrm{H}, \mathrm{H}-8), 7.86(\mathrm{~s}, 1 \mathrm{H}, \mathrm{H}-2), 8.01(\mathrm{~d}$, $1 \mathrm{H}, J=7.8 \mathrm{~Hz}, \mathrm{H}-7), 9.76(\mathrm{dd}, 1 \mathrm{H}, J=8.3,1.4 \mathrm{~Hz}, \mathrm{H}-10), 10.21$ $(\mathrm{s}, 1 \mathrm{H}, \mathrm{H}-5){ }^{13} \mathrm{C}-\mathrm{NMR}(75 \mathrm{MHz}, \mathrm{CDCl}, \delta \mathrm{ppm}): 14.3(\mathrm{Me})$, $56.2(2 \mathrm{MeO}), 60.9\left(\mathrm{CH}_{3}\right), 110.3\left(\mathrm{CH}^{\prime}\right), 110.5,111.9(\mathrm{CH})$, $120.1,123.7,124.1(\mathrm{CH}), 127.7(\mathrm{CH}), 128.3(\mathrm{CH}), 128.4$ $(\mathrm{CH}), 129.3(\mathrm{CH}), 131.1(\mathrm{CH}), 131.5,136.5,138.5(\mathrm{CH})$, 141.1, 149.3, 153.1, 164.0 (COO), 184.4 (CO).IR ( $\mathrm{KBr}, \mathrm{Cm}^{-}$ 1): 3116, 1714, 1614, 1578, 1516, 1453, 1411, 1351, 1324, 1268, 1189, 1118, 1091. Anal. Calcd. for $\mathrm{C}_{23} \mathrm{H}_{2} \mathrm{~N}_{2} \mathrm{O}$ (404.43): C, 68.31; $\mathrm{H}, 4.98 ; \mathrm{N}, 6.93$. Found: $\mathrm{C}, 68.57 ; \mathrm{H}^{2}$, $5.13 ; \mathrm{N}, 7.22$.

1-Benzoyl-3-(3,4-dimethoxybenzoyl)pyrrolo[1,2c]quinazoline (7). Pale yellow crystals (from $\mathrm{CHCl}_{3}$ Et O). ${ }^{1} \mathrm{H}-\mathrm{NMR}\left(300 \mathrm{MHz}, \mathrm{CDCl}{ }_{3}, \delta \mathrm{ppm}\right): 3.88,4.01$ (s, 6H, 2MeO), $6.84\left(\mathrm{~d}, 1 \mathrm{H}, J=8.2, \mathrm{H}-5^{3}\right), 7.39-7.45(\mathrm{~m}, 4 \mathrm{H} \mathrm{Ar}) 7.42$ (s, $1 \mathrm{H}, \mathrm{H}-2), 7.44-7.53(\mathrm{~m}, 3 \mathrm{H}, \mathrm{H}-9, \mathrm{Ph}), \mathbf{7 . 6 4 - 7 . 7 0}(\mathrm{m}, \mathrm{H}$,
$\mathrm{H}-8), 7.83-7.88(\mathrm{~m}, 2 \mathrm{H}$, ortho-Ph), $7.94(\mathrm{~d}, 1 \mathrm{H}, J=7.8 \mathrm{~Hz}, \mathrm{H}-$ 7), $8.86(\mathrm{dd}, 1 \mathrm{H}, J=8.3,1.4 \mathrm{~Hz}, \mathrm{H}-10), 10.28(\mathrm{~s}, 1 \mathrm{H}, \mathrm{H}-5) .{ }^{13} \mathrm{C}-$ NMR (75 MHz, CDCl,$\delta$ ppm): 56.0, 56.1 (2MeO), 110.3 (C5), $110.5,111.9(\mathrm{C}-2), 117.6,120.0,123.9,126.2(\mathrm{CH}), 128.2$ (CH), 128.5(CH), $128.6(\mathrm{CH}), 129.4(\mathrm{CH}), 129.8(\mathrm{CH}), 131.2$ (CH), 131.4, $132.8(\mathrm{CH}), 136.1,138.4(\mathrm{CH}), 139.2,141.0$, $149.2,153.1,184.2$ (CO), $191.8(\mathrm{CO})$. IR $\left(\mathrm{KBr}, \mathrm{cm}^{-1}\right)$ : 3079, 2959, 1628, 1607, 1577, 1512, 1450, 1402, 1357, 1325, $1263,1213,1174,1142,1119,1020$. Anal. Calcd. for $\mathrm{C}_{2} \mathrm{H}_{2} \mathrm{~N}_{2} \mathrm{O}$ (436.47): $\mathrm{C}, 74.30 ; \mathrm{H}, 4.62 ; \mathrm{N}, 6.42$. Found: $\mathrm{C}, 74.48 ; \mathrm{H}^{4}, 4.75 ; \mathrm{N}, 6.29$

Methyl 3-(1-naphthoyl)pyrrolo[1,2-c]quinazoline-1carboxylate (8). Beige crystals (from $\mathrm{CHCl}$, EtO ${ }^{1}{ }^{1} \mathrm{H}-\mathrm{NMR}$ (300 MHz, CDCl,$\delta \mathrm{ppm}): 3.86(\mathrm{~s}, 3 \mathrm{H}, \mathrm{Me})$, 7.57-7.63 (m, $3 \mathrm{H}$, naphth), 7.64 (s, $1 \mathrm{H}, \mathrm{H}-2), 7.68-7.85(\mathrm{~m}, \mathrm{H}-7, \mathrm{H}-8, \mathrm{H}-9)$, 7.94-7.98 (m, 1H, naphth), 8.05-8.09 ( $\mathrm{m}, 2 \mathrm{H}$, naphth) 8.178.21 ( $m, 1 \mathrm{H}, \mathrm{H}-2^{\prime}$, naphth), $9.76(\mathrm{dd}, 1 \mathrm{H}, J=8.3,1.4 \mathrm{~Hz}, \mathrm{H}-$ 10), $10.62(\mathrm{~s}, 1 \mathrm{H}, \mathrm{H}-5) .{ }^{13} \mathrm{C}-\mathrm{NMR}(75 \mathrm{MHz}, \mathrm{CDCl}, \delta \mathrm{ppm})$ : $51.9(\mathrm{Me}), 110.4,120.0,124.4(\mathrm{CH}), 124.8,125.2(\mathrm{CH}), 126.6$ (CH), 127.2(CH), $127.4(\mathrm{CH}), 127.8(\mathrm{CH}), 128.4(\mathrm{CH}), 128.5$ (2CH), 130.7, $131.2(\mathrm{CH}), 131.4(\mathrm{CH}), 131.5(\mathrm{CH}), 133.8$, $136.6,137.2,138.6$ (CH), 141.3, 164.1 (COO), 187.1 (CO). IR $\left(\mathrm{KBr}, \mathrm{Cm}^{-1}\right): 3095,1716,1620,1535,1475,1455,1400$, $1349,1319,1233,1199,1184,1145,1093,1046$. Anal. Calcd. for $\mathrm{C}_{2} \mathrm{H}_{16} \mathrm{~N}_{2} \mathrm{O}_{3}$ (380.41): C, 75.78; $\mathrm{H}, 4.24 ; \mathrm{N}, 7.36$. Found: $\mathrm{C}, 76.03 ; \mathrm{H}, 4.45 ; \mathrm{N}, 7.51$.

Ethyl 3-(1-naphthoyl)pyrrolo[1,2-c]quinazoline-1carboxylate (9). Pale yellow crystals (from CHCl,/Et O). ${ }^{1} \mathrm{H}$ $\operatorname{NMR}\left(300 \mathrm{MHz}, \mathrm{CDCl}_{3}, \delta \mathrm{ppm}\right): 1.33(\mathrm{t}, 3 \mathrm{H}, J=7.1 \mathrm{~Hz}, \mathrm{Me})$, $4.36\left(q, 2 \mathrm{H}, J=7.1 \mathrm{~Hz}^{2} \mathrm{CH}_{2}\right), 7.55-7.62$ (m, 3H, naphth), 7.65 (s, $1 \mathrm{H}, \mathrm{H}-2), 7.67-7.83$ (m, H-7, H-8, H-9), 7.94-7.98 ( $\mathrm{m}, 1 \mathrm{H}$, naphth), 8.04-8.08 ( $\mathrm{m}, 2 \mathrm{H}$, naphth) 8.18-8.23 ( $\mathrm{m}$, $1 \mathrm{H}, \mathrm{H}-2$ ', naphth), 9.74 (dd, $1 \mathrm{H}, J=8.3,1.4 \mathrm{~Hz}, \mathrm{H}-10$ ), 10.60 (s, $1 \mathrm{H}, \mathrm{H}-5) .{ }^{13} \mathrm{C}-\mathrm{NMR}(75 \mathrm{MHz}, \mathrm{CDCl}, \delta \mathrm{ppm}): 14.3(\mathrm{Me})$, $60.9\left(\mathrm{CH}_{2}\right), 111.0,120.0,124.3(\mathrm{CH}), 124.8,125.3(\mathrm{CH})$, $126.6(\mathrm{CH}), 127.3(\mathrm{CH}), 127.4(\mathrm{CH}), 127.9(\mathrm{CH}), 128.3(\mathrm{CH})$, $128.4(\mathrm{CH}), 128.5(\mathrm{CH}), 130.8,131.0(\mathrm{CH}), 131.3(\mathrm{CH}), 131.4$ (CH), 133.8, 136.6, 137.1, 138.6 (CH), 141.3, 163.7 (COO), 187.0 (CO). IR (KBr, $\left.\mathrm{Cm}^{-1}\right)$ : 3051, 1711, 1628, 1565, 1480, 1451, 1401, 1382, 1319, 1277, 1210, 1171, 1123, 1089 Anal. Calcd. for $\mathrm{C}_{25} \mathrm{H}_{13} \mathrm{~N}_{2} \mathrm{O}_{3}$ (394.43): C, 76.13; $\mathrm{H}, 4.60 ; \mathrm{N}, 7.10$. Found: $\mathrm{C}, 76.40 ; \mathrm{H}^{2}, 4.85 ; \mathrm{N}, 7.29$.

1-Acetyl-3-(1-naphthoyl)pyrrolo[1,2-c]quinazoline (10). Pale yellow crystals (from CHCl,/EtO). ${ }^{1} \mathrm{H}-\mathrm{NMR}$ (300 $\left.\mathrm{MHz} \mathrm{CDCl}_{3}, \delta \mathrm{ppm}\right): 2.54(\mathrm{~s}, 3 \mathrm{H}, \mathrm{Me}), 7.56(\mathrm{~s}, 1 \mathrm{H}, \mathrm{H}-2)$, 7.57-7.63 ( $\mathrm{m}, 3 \mathrm{H}$, naphth), 7.68-7.74 ( $\mathrm{m}, 1 \mathrm{H}, \mathrm{H}-9), 7.76$ (dd, $1 \mathrm{H}, J=8.4,1.4 \mathrm{~Hz}, \mathrm{H}-7), 7.81-7.87(\mathrm{~m}, 1 \mathrm{H}, \mathrm{H}-8), 7.95-$ 7.99 ( $\mathrm{m}, 1 \mathrm{H}$, naphth), 8.05-8.09 (m, 2H, naphth) 8.18-8.22 ( $m, 1 \mathrm{H}, \mathrm{H}-2^{\prime}$, naphth), 9.72 (dd, $\left.1 \mathrm{H}, J=8.4,1.4 \mathrm{~Hz}, \mathrm{H}-10\right)$, 10.63 (s, 1H, H-5). ${ }^{13} \mathrm{C}-\mathrm{NMR}\left(75 \mathrm{MHz}, \mathrm{CDCl}_{3}, \delta \mathrm{ppm}\right): 29.9$ (Me), 119.4, 120.2, 124.3 (CH), 124.8, 125.2(CH), 126.7 $(\mathrm{CH}), 127.2(\mathrm{CH}), 127.5(\mathrm{CH}), 128.0(\mathrm{CH}), 128.4(\mathrm{CH}), 128.4$ (CH), $128.5(\mathrm{CH}), 130.8,131.0(\mathrm{CH}), 131.5(\mathrm{CH}), 131.8(\mathrm{CH})$, 133.8, 136.6, $138.4(\mathrm{CH}), 141.6,186.9$ (CO), 193.4 (COMe). IR $\left(\mathrm{KBr}, \mathrm{Cm}^{-1}\right): 3106,1666,1627,1522,1472,1447,1400$, 1322, 1266, 1179, 1133. Anal. Calcd. for $\mathrm{C}_{2} \mathrm{H}_{1} \mathrm{~N}_{2} \mathrm{O}$ (364.41): C, 79.11; $\mathrm{H}, 4.43 ; \mathrm{N}, 7.69$. Found: $\mathrm{C}, 79.34 ; \mathrm{H}^{2}$, 4.75; N, 7.87.

Methyl 3-(2-naphthoyl)pyrrolo[1,2-c]quinazoline-1carboxylate (11). White crystals (from CHCl, Et $\mathrm{O}) .{ }^{1} \mathrm{H}$ -

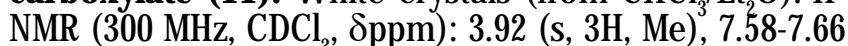
(m, 3H, naphth), 7.67-7.74 (m, 1H, H-9), 7.77-7.82 (m, $1 \mathrm{H}$, $\mathrm{H}-8$ ), 7.89 (s, $1 \mathrm{H}, \mathrm{H}-2$ ), 7.94-8.05 (m, 4H, H-7, naphth), 8.40 (d, $1 \mathrm{H}, J=1.4 \mathrm{~Hz}$, naphth), 9.76 (dd, $1 \mathrm{H}, J=8.2,1.5 \mathrm{~Hz}, \mathrm{H}$ 10), $10.38(\mathrm{~s}, 1 \mathrm{H}, \mathrm{H}-5){ }^{13} \mathrm{C}-\mathrm{NMR}(75 \mathrm{MHz}, \mathrm{CDCl}, \delta \mathrm{ppm})$ : $52.0(\mathrm{Me}), 110.3,120.0,123.7,125.3(\mathrm{CH}), 127.0\left(\mathrm{CH}^{\prime} \mathrm{H}\right), 127.7$ $(\mathrm{CH}), 127.9(\mathrm{CH}), 128.31(\mathrm{CH}), 128.37(\mathrm{CH}), 128.4(\mathrm{CH})$, $128.6(\mathrm{CH}), 129.3(\mathrm{CH}), 130.3(\mathrm{CH}), 130.5(\mathrm{CH}), 131.3(\mathrm{CH})$, 
$132.3,135.2,136.2,136.9,138.4(\mathrm{CH}), 141.2,164.2$ (COO),

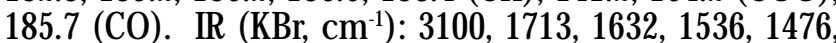
$1454,1402,1324,1250,1202,1183$, 1091.Anal. Calcd. for $\mathrm{C}_{2} \mathrm{H}^{\mathrm{N}} \mathrm{O}$ (380.41): $\mathrm{C}, 75.78 ; \mathrm{H}, 4.24 ; \mathrm{N}, 7.36$. Found: $\mathrm{C}$, $75.97 ; \mathrm{H}, 4.60 ; \mathrm{N}, 7.49$.

Ethyl 3-(2-naphthoyl)pyrrolo[1,2-c]quinazoline-1carboxylate (12). Beige crystals (from CHCl/EtO). ${ }^{1} \mathrm{H}-$ NMR ( $300 \mathrm{MHz}, \mathrm{CDCl}, \delta p p m): 1.39(\mathrm{t}, 3 \mathrm{H}, J=7.1 \mathrm{~Hz}, \mathrm{Me})$, $4.41\left(\mathrm{q}, 2 \mathrm{H}, J=7.1 \mathrm{~Hz}, \mathrm{CH}_{2}\right), 7.58-7.66$ (m, 3H, naphth), 7.68-7.73 (m, 1H, H-9), 7.77-7.82 (m, 1H, H-8), $7.89(\mathrm{~s}, 1 \mathrm{H}$, $\mathrm{H}-2$ ), 7.94-8.05 (m, 4H, H-7, naphth), 8.41 (d, $1 \mathrm{H}, J=1.4 \mathrm{~Hz}$, naphth), $9.76(\mathrm{dd}, 1 \mathrm{H}, J=8.2,1.5 \mathrm{~Hz}, \mathrm{H}-10), 10.38(\mathrm{~s}, 1 \mathrm{H}, \mathrm{H}-$ 5). ${ }^{13} \mathrm{C}-\mathrm{NMR}(75 \mathrm{MHz}, \mathrm{CDCl}, \delta \mathrm{ppm}): 14.3(\mathrm{Me}), 60.9\left(\mathrm{CH}_{2}\right)$, $110.8,120.1,123.8,125.3(\mathrm{CH}), 127.0 \mathrm{CH}), 127.8(\mathrm{CH})$, $127.9(\mathrm{CH}), 128.3(\mathrm{CH}), 128.34(\mathrm{CH}), 128.5(\mathrm{CH}), 128.6$ (CH), $129.4(\mathrm{CH}), 130.1(\mathrm{CH}), 130.5(\mathrm{CH}), 131.2(\mathrm{CH}), 132.5$, 135.3, 136.3, 136.8, 138.5 (CH), 141.2, 163.9 (COO), 185.6 (CO). IR (KBr, $\left.\mathrm{Cm}^{-1}\right): 3105,1705,1631,1534,1475,1451$, $1405,1353,1323,1249,1197,1182,1089,1036$. Anal. Calcd. for $\mathrm{C}_{25} \mathrm{H}_{18} \mathrm{~N}_{2} \mathrm{O}_{3}$ (394.43): C, 76.13; $\mathrm{H}, 4.60 ; \mathrm{N}, 7.10$. Found: $\mathrm{C}, 76.21 ; \mathrm{H}^{2}, 4.77 ; \mathrm{N}, 7.31$.

1-Benzoyl-3-(2-naphthoyl)pyrrolo[1,2-c]quinazoline (13). Pale yellow crystals (from CHCl//EtO). ${ }^{H} \mathrm{H}-\mathrm{NMR}$ (300 $\left.\mathrm{MHz}, \mathrm{CDCl}_{3}, \delta \mathrm{ppm}\right)$ : 7.46-7.51 (m, 2H,Ph), 7.55-7.64 (m, $4 \mathrm{H}, \mathrm{H}-9$, naphth), 7.56 (s, $1 \mathrm{H}, \mathrm{H}-2), 7.75-7.81$ ( $\mathrm{m}, 1 \mathrm{H}, \mathrm{H}-8)$, 7.89-7.96 ( $\mathrm{m}, 6 \mathrm{H}, \mathrm{Ph}$, naphth), 8.05 (dd, $1 \mathrm{H}, \mathrm{dd}, 1 \mathrm{H}, J=8.2$, $1.4 \mathrm{~Hz}, \mathrm{H}-7$ ), 8.37 (d, $1 \mathrm{H}, J=1.4 \mathrm{~Hz}$, naphth), 8.96 (dd, $1 \mathrm{H}$, $J=8.2,1.5 \mathrm{~Hz}, \mathrm{H}-10), 10.43(\mathrm{~s}, 1 \mathrm{H}, \mathrm{H}-5) .{ }^{13} \mathrm{C}-\mathrm{NMR}(75 \mathrm{MHz}$, $\left.\mathrm{CDCl}_{3}, \delta \mathrm{ppm}\right): 117.8,119.9,123.4,125.2(\mathrm{CH}), 126.3(\mathrm{CH})$, $127.0^{\prime}(\mathrm{CH}), 127.8(\mathrm{CH}), 128.3(\mathrm{CH}), 128.4(\mathrm{CH}), 128.5(\mathrm{CH})$, $128.6(\mathrm{CH}), 128.7(\mathrm{CH}), 129.9(\mathrm{CH}), 130.5(\mathrm{CH}), 130.7(\mathrm{CH})$ 131.3, $131.4(\mathrm{CH}), 132.3,133.0(\mathrm{CH}), 135.2,136.1,138.4$ (CH), 138.9, 141.1, 185.6 (CO), $191.9(\mathrm{CO})$. IR $\left(\mathrm{KBr}, \mathrm{Cm}^{-1}\right)$ : $3051,1647,1620,1524,1474,1450,1402,1356,1327$, $1271,1213,1187,1109,1018$. Anal. Calcd. for $\mathrm{C}_{1} \mathrm{H}_{1} \mathrm{~N}_{2} \mathrm{O}$ (426.48): $\mathrm{C}, 81.67 ; \mathrm{H}, 4.25 ; \mathrm{N}, 6.57$. Found: $\mathrm{C}, 81.96 ;{ }^{2} \mathrm{H}^{2}$, 4.47; N, 6.82

Ethyl 8-fluoro-3-(2-naphthoyl)pyrrolo[1,2c]quinazoline-1-carboxylate (14). White crystals (from $\mathrm{CHCl} /$ Et O). ${ }^{-} \mathrm{H}-\mathrm{NMR}(300 \mathrm{MHz}, \mathrm{CDCl}, \delta \mathrm{ppm}): 1.36(\mathrm{t}, 3 \mathrm{H}$, $J=7.1 \mathrm{~Hz}, \mathrm{Me}), 4.40\left(\mathrm{q}, 2 \mathrm{H}, J=7.1 \mathrm{~Hz}, \mathrm{CH}_{2}\right), 7.40-7.47(\mathrm{~m}$, 1H, H-9), 7.59-7.70 (m, 3H, H-7, 2H-naphth), 7.89 (s, 1H, H2), 7.95-8.03 (m, 4H, naphth), 8.40 (bs, $1 \mathrm{H}, \mathrm{H}-2 /$ naphth), $9.88(\mathrm{dd}, 1 \mathrm{H}, J=9.2,6.3 \mathrm{~Hz}, \mathrm{H}-10), 10.38(\mathrm{~s}, 1 \mathrm{H}, \mathrm{H}-5) .{ }^{13} \mathrm{C}-$ $\operatorname{NMR}\left(75 \mathrm{MHz}, \mathrm{CDCl}_{2}, \delta \mathrm{ppm}\right): 14.4(\mathrm{Me}), 61.1\left(\mathrm{CH}_{2}\right), 103.4$ $110.4,113.7(\mathrm{~d}, J=21.7 \mathrm{~Hz}, \mathrm{C}-7), 116.7,117.0\left(\mathrm{~d}^{2}, J=22.8\right.$ $\mathrm{Hz}, \mathrm{C}-9), 123.7,125.3(\mathrm{CH}), 127.1(\mathrm{CH}), 128.0(\mathrm{CH}), 128.4$ $(\mathrm{CH}), 128.7(\mathrm{CH}), 129.4(\mathrm{CH}), 129.6,130.3(\mathrm{CH}), 130.6$ (CH), 132.5, 135.3, 136.2, 136.5, $139.5(\mathrm{CH}), 143.2(\mathrm{~d}, \mathrm{~J}=$ $12.6 \mathrm{~Hz}, \mathrm{C}-6 \mathrm{a}), 163.7$ (d, J= 253.0 Hz, C-8), 163.9 (COO), 185.7 (CO).IR $\left(\mathrm{KBr}, \mathrm{Cm}^{-1}\right)$ : 3107, 1703, 1667, 1600, 1534, $1458,1373,1350,1298,1260,1226,1183,1155$, 1091. Anal. Calcd. for $\mathrm{C}_{23} \mathrm{H}_{7} \mathrm{FN}_{2} \mathrm{O}$ ( 428.88$)$ : C, 72.81; $\mathrm{H}, 4.15 ; \mathrm{N}, 6.79$. Found: $\mathrm{C}, 73.12 ; \mathrm{H}, 4.34 ; \mathrm{N}, 6.96$.

1,3-Dibenzoyl-8-chloropyrrolo[1,2-c]quinazoline (15). Orange crystals (from CHCl//Et, O). ${ }^{2} \mathrm{H}-\mathrm{NMR}(300 \mathrm{MHz}$, $\mathrm{CDCl}_{2}$, Sppm): 7.42-7.67 (m, 8H, $\left.\mathrm{H}-2, \mathrm{H}-9, \mathrm{Ph}\right), 7.86-7.92$ $(\mathrm{m}, 4 \mathrm{H}, \mathrm{Ph}), 7.98(\mathrm{~d}, 1 \mathrm{H}, J=2.2 \mathrm{~Hz}, \mathrm{H}-7), 8.94(\mathrm{~d}, 1 \mathrm{H}, J=9.0$ $\mathrm{Hz}, \mathrm{H}-10), 10.37$ (s, 1H, H-5). ${ }^{13} \mathrm{C}-\mathrm{NMR}$ ( $75 \mathrm{MHz}, \mathrm{CDCl}_{\text {, ä }}$ ppm): $117.8,118.4,123.3,127.7(\mathrm{CH}), 128.0(\mathrm{CH}), 128.6$ (CH), 128.8(CH), $129.2(\mathrm{CH}), 129.8(\mathrm{CH}), 130.5(\mathrm{CH}), 132.5$ $(\mathrm{CH}), 133.1(\mathrm{CH}), 135.8,137.0,138.6,138.7,139.3(\mathrm{CH})$,

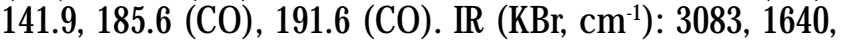
$1623,1597,1523,1446,1399,1349,1319,1295,1234$, 1211, 1177, 1078. Anal. Calcd. for $\mathrm{C}_{2} \mathrm{H}_{1} \mathrm{CIN}_{2} \mathrm{O}$ (410.86): C, 73.08; H, 3.68; N, 8.63. Found: C, 73.39; H, 3.85; N, 8.82.

Ethyl 3-(3-nitrobenzoyl)-8-chloropyrrolo[1,2c]quinazoline-1-carboxylate (16). Pale yellow crystals (from $\left.\mathrm{CHCl}_{3}\right) .{ }^{1} \mathrm{H}-\mathrm{NMR}\left(300 \mathrm{MHz}, \mathrm{CDCl}_{3}+\mathrm{TFA}, \delta \mathrm{ppm}\right): 1.47$ (t, $3 \mathrm{H}, J=7.1 \mathrm{~Hz}, \mathrm{Me}), 4.47\left(\mathrm{q}, 2 \mathrm{H}, J=7.1 \mathrm{~Hz}, \mathrm{CH}_{2}\right), 7.87$ (t, $\left.1 \mathrm{H}, J=8.0 \mathrm{~Hz}, \mathrm{H}-5^{\prime}\right), 7.92(\mathrm{dd}, 1 \mathrm{H}, J=9.0,2.0 \mathrm{~Hz}, \mathrm{H}-9), 7.99$ $(\mathrm{s}, 1 \mathrm{H}, \mathrm{H}-2), 8.15(\mathrm{~d}, 1 \mathrm{H}, J=2.0 \mathrm{~Hz}, \mathrm{H}-7), 8.23-8.27(\mathrm{~m}, 1 \mathrm{H}$, $\left.\mathrm{H}-4^{\prime}\right), 8.58-8.62\left(\mathrm{~m}, 1 \mathrm{H}, \mathrm{H}-6^{\prime}\right), 8.73\left(\mathrm{t}, 1 \mathrm{H}, J=1.9 \mathrm{~Hz}, \mathrm{H}-2^{\prime}\right)$, $9.86(\mathrm{~d}, 1 \mathrm{H}, \mathrm{J}=9.0 \mathrm{~Hz}, \mathrm{H}-10), 11.06(\mathrm{~s}, 1 \mathrm{H}, \mathrm{H}-5) .{ }^{13} \mathrm{C}-\mathrm{NMR}$ (75 MHz, CDCl + TFA, $\delta$ ppm): $14.0(\mathrm{Me}), 62.9\left(\mathrm{CH}_{3}\right), 114.1$, $117.6,122.5(\mathrm{CH}), 124.1(\mathrm{CH}), 124.6,127.9(\mathrm{CH}), 130.0$ (CH), $130.5(\mathrm{CH}), 131.9(\mathrm{CH}), 133.0(\mathrm{CH}), 133.7(\mathrm{CH}), 134.8$ (CH), 135.8, 138.4, 140.3, 142.8, 148.3, 163.2 (COO), 183.7 (CO). IR $\left(\mathrm{KBr}_{\mathrm{Cm}} \mathrm{Cm}^{-1}\right)$ 3091, 1713, 1637, 1536, 1455, 1347, 1229, 1196, 1153, 1099, 1080. Anal. Calcd. for $\mathrm{C}_{21} \mathrm{H}_{1} \mathrm{CIN}_{3} \mathrm{O}_{5}$ (423.82): C, 59.52; $\mathrm{H}, 3.33$; N, 9.91. Found: $\mathrm{C}^{21}{ }^{49} .61 ;{ }^{3} \mathrm{H}^{5}$, 3.72; N, 10.22 .

1-Benzoyl-3-(3-nitrobenzoyl)-8-chloropyrrolo[1,2c] quinazoline (17). Yellow crystals (from $\mathrm{CHCl}_{3}$ ). ${ }^{1} \mathrm{H}-\mathrm{NMR}$ ( $\left.300 \mathrm{MHz}, \mathrm{CDCl}_{3}+\mathrm{TFA}, \delta \mathrm{ppm}\right): 7.51-7.63(\mathrm{~m}, 4 \mathrm{H}, \mathrm{H}-9,3 \mathrm{H}-$ $\mathrm{Ph}), 7.51(\mathrm{~s}, 1 \mathrm{H}, \mathrm{H}-2), 7.73\left(\mathrm{t}, 1 \mathrm{H}, J=8.0 \mathrm{~Hz}, m-\mathrm{PhNO}_{2}\right)$, 7.91-7.95 (m, 2H-Ph), $8.04(\mathrm{~d}, 1 \mathrm{H}, J=2.0 \mathrm{~Hz}, \mathrm{H}-7), 8.18-$ $8.22\left(\mathrm{~m}, 1 \mathrm{H}, p-\mathrm{PhNO}_{2}\right), 8.41-8.46\left(\mathrm{~m}, 1 \mathrm{H}, \mathrm{m}-\mathrm{PhNO}_{2}\right), 8.72$ $(\mathrm{t}, 1 \mathrm{H}, J=8.0 \mathrm{~Hz}, 0-\mathrm{PhNO}$ ), $9.01(\mathrm{~d}, 1 \mathrm{H}, J=9.0 \mathrm{~Hz}, \mathrm{H}-10)$, $10.38(\mathrm{~s}, 1 \mathrm{H}, \mathrm{H}-5) .{ }^{13} \mathrm{C}-\mathrm{NMR}(75 \mathrm{MHz}, \mathrm{CDCl} 3+\mathrm{TFA}, \delta \mathrm{ppm})$ : $118.2,118.3,122.4,124.1(\mathrm{CH}), 126.8(\mathrm{CH}), 128.0(\mathrm{CH})$, $128.1(\mathrm{CH}), 128.7(\mathrm{CH}), 129.1(\mathrm{CH}), 129.9(\mathrm{CH}), 130.1(\mathrm{CH})$, $131.0(\mathrm{CH}), 133.3(\mathrm{CH}), 134.6(\mathrm{CH}), 136.7,137.7,138.6$, 140.0 (CH), 142.1, 148.3, 182.7 (CO), 191.2 (CO). IR ( $\mathrm{KBr}$, $\left.\mathrm{cm}^{-1}\right)$ : 3078, 1651, 1630, 1598, 1529, 1452, 1348, 1262, 1203, 1181, 1149, 1082. Anal. Calcd. for $\mathrm{C}_{25} \mathrm{H}_{1} \mathrm{CIN}_{3} \mathrm{O}$ (455.86): C, 65.87; $\mathrm{H}, 3.10 ; \mathrm{N}, 9.22$. Found: $\mathrm{C}^{2}, 66.08 ;{ }^{3} \mathrm{H}^{4}$ 3.37; N, 9.42.

Ethyl 3-(4-nitrobenzoyl)-8-chloropyrrolo[1,2c] quinazoline-1-carboxylate (18). Yellow crystals (from $\mathrm{CHCl}_{3}$ ). ${ }^{1} \mathrm{H}-\mathrm{NMR}(300 \mathrm{MHz}, \mathrm{CDCl}+\mathrm{TFA}, \delta \mathrm{ppm}): 1.46(\mathrm{t}$, $3 \mathrm{H}, J=7.1 \mathrm{~Hz}, \mathrm{Me}), 4.49\left(\mathrm{q}, 2 \mathrm{H}, J=7.1 \mathrm{~Hz}, \mathrm{CH}_{2}\right), 7.85(\mathrm{dd}$, $1 \mathrm{H}, \mathrm{J}=9.0,2.0 \mathrm{~Hz}, \mathrm{H}-9), 7.99(\mathrm{~s}, 1 \mathrm{H}, \mathrm{H}-2), 8.05(\mathrm{~d}, 2 \mathrm{H}, J=8.9$ $\left.\mathrm{Hz}, \mathrm{H}-2^{\prime}, \mathrm{H}-6^{\prime}\right), 8.12(\mathrm{~d}, 1 \mathrm{H}, J=2.0 \mathrm{~Hz}, \mathrm{H}-7), 8.47(\mathrm{~d}, 2 \mathrm{H}$, $\left.J=8.9 \mathrm{~Hz}, \mathrm{H}-3^{\prime}, \mathrm{H}-5^{\prime}\right), 9.86(\mathrm{~d}, 1 \mathrm{H}, J=9.0 \mathrm{~Hz}, \mathrm{H}-10), 10.92$ (s, 1H, H-5). ${ }^{13} \mathrm{C}-\mathrm{NMR}(75 \mathrm{MHz}, \mathrm{CDCl}+\mathrm{TFA}, \delta \mathrm{ppm}): 14.2$ (Me), $62.6\left(\mathrm{CH}_{2}\right), 113.6,117.8,123.5(\mathrm{CH}), 124.2(\mathrm{CH})$, $124.3,130.0(\mathrm{CH}), 130.2(\mathrm{CH}), 131.4(\mathrm{CH}), 132.8(\mathrm{CH})$, $135.5,136.1,139.8,142.2,142.4,150.4,163.1$ (COO), 184.2 (CO). IR $\left(\mathrm{KBr}_{\mathrm{Cm}} \mathrm{Cm}^{-1}\right)$ : 3093, 2970, 1718, 1641, 1534, 1472, $1449,1348,1298,1225,1180,1152,1076$. Anal. Calcd. for $\mathrm{C}_{2} \mathrm{H}_{1} \mathrm{ClN}_{3} \mathrm{O}_{5}$ (423.82): C, 59.52; $\mathrm{H}, 3.33 ; \mathrm{N}, 9.91$. Found: $\mathrm{C}$, 59.77; $\mathrm{H}, 3.58 ; \mathrm{N}, 10.16$.

1-Benzoyl-3-(4-nitrobenzoyl)-8-chloropyrrolo[1,2c]quinazoline (19). Yellow crystals from $\mathrm{CHCl}_{3}{ }^{1}{ }^{1} \mathrm{H}-\mathrm{NMR}$ $(300 \mathrm{MHz}, \mathrm{CDCl}+\mathrm{TFA}, \delta \mathrm{ppm}): 7.55-7.61(\mathrm{~m}, 2 \mathrm{H}, \mathrm{Ph}), 7.63$ ( $\mathrm{s}, 1 \mathrm{H}, \mathrm{H}-2), 7.72$ (dd, $1 \mathrm{H}, J=9.0,2.0 \mathrm{~Hz}, \mathrm{H}-9), 7.89-9.93(\mathrm{~m}$, $2 \mathrm{H}, \mathrm{Ph}), 8.04\left(\mathrm{~d}, 2 \mathrm{H}, J=8.9 \mathrm{~Hz}, \mathrm{H}-2^{\prime}, \mathrm{H}-6^{\prime}\right), 8.15(\mathrm{~d}, 1 \mathrm{H}$, $J=9.0,2.0 \mathrm{~Hz}, \mathrm{H}-7), 8.41\left(\mathrm{~d}, 2 \mathrm{H}, J=8.9 \mathrm{~Hz}, \mathrm{H}-3^{\prime}, \mathrm{H}-5^{\prime}\right), 8.84$ $(\mathrm{d}, 1 \mathrm{H}, \mathrm{J}=2.0 \mathrm{~Hz}, \mathrm{H}-10), 10.84(\mathrm{~s}, 1 \mathrm{H}, \mathrm{H}-5) .{ }^{13} \mathrm{C}-\mathrm{NMR}(75$ $\left.\mathrm{MHz}, \mathrm{CDCl}_{3}+\mathrm{TFA}, \delta \mathrm{ppm}\right): 117.8,119.3,123.8,124.1(\mathrm{CH})$, $125.0(\mathrm{CH}), 128.1(\mathrm{CH}), 129.2(\mathrm{CH}), 130.1(\mathrm{CH}), 130.9(\mathrm{CH})$, $132.2(\mathrm{CH}), 134.6(\mathrm{CH}), 136.0,137.4,137.6,139.3,141.4$, $141.5,142.7,150.2,183.9(\mathrm{CO}), 192.6$ (CO). IR (KBr, $\mathrm{Cm}^{-}$ 1): $3105,1648,1628,1599,1525,1450,1349,1258,1208$, $1178,1155,1079$, 1019. Anal. Calcd. for $\mathrm{C}_{25} \mathrm{H}_{1} \mathrm{CIN}_{3} \mathrm{O}_{4}$ (455.86): C, 65.87; $\mathrm{H}, 3.10 ; \mathrm{N}, 9.22$. Found: $\mathrm{C}^{25}, 66.11 ; \mathrm{H}^{4}$, 3.29; N, 9.38 .

1-Benzoyl-8-chloro-3-(1-naphthoyl) pyrrolo[1,2c] quinazoline (20). Yellow crystals (from CHCl $/ \mathrm{EtO}) .{ }^{1} \mathrm{H}$ NMR ( $\left.300 \mathrm{MHz}, \mathrm{CDCl}_{3}, \delta \mathrm{ppm}\right)$ : 7.30-7.37 (m, 2H, $\left.\mathrm{Ph}\right), 7.42-$ 7.51 ( $\mathrm{m}, 6 \mathrm{H}, \mathrm{H}-2, \mathrm{H}-9$, Ph, naphth), 7.65 (dd, $1 \mathrm{H}, \mathrm{J}=7.0,1.1$ $\mathrm{Hz}$, naphth), 7.72-7.75 (m, $2 \mathrm{H}, \mathrm{Ph}), 7.82-7.86(\mathrm{~m}, 1 \mathrm{H}$, napth) $7.92(\mathrm{~d}, 1 \mathrm{H}, J=8.2 \mathrm{~Hz}$, naphth), $7.98(\mathrm{~d}, 1 \mathrm{H}, J=2.1 \mathrm{~Hz}, \mathrm{H}-7)$, 8.12-8.17 ( $\mathrm{m}, 1 \mathrm{H}, J=1.5 \mathrm{~Hz}$, naphth), $8.89(\mathrm{~d}, 1 \mathrm{H}, J=9.0, \mathrm{H}-$ 10), $10.57(\mathrm{~s}, 1 \mathrm{H}, \mathrm{H}-5){ }^{13} \mathrm{C}-\mathrm{NMR}(75 \mathrm{MHz}, \mathrm{CDCl}, \delta \mathrm{ppm})$ : $118.1,118.6,124.8(\mathrm{CH}), 125.2(\mathrm{CH}), 125.5(\mathrm{CH}), 127.5$ $(\mathrm{CH}), 127.8(\mathrm{CH}), 128.0(\mathrm{CH}), 128.4(\mathrm{CH}), 128.6(\mathrm{CH}), 128.7$ 
$(\mathrm{CH}), 128.8(\mathrm{CH}), 128.9(\mathrm{CH}), 129.9(\mathrm{CH}), 130.1(\mathrm{CH}), 130.8$ (CH), 131.1, 131.6, 132.0, 133.9, 136.3, 136.4, 137.4, 138.5, $138.6(\mathrm{CH}), 139.4(\mathrm{CH}), 140.6(\mathrm{CH}), 142.2(\mathrm{CH}), 187.8(\mathrm{CO})$, $191.6(\mathrm{CO}) . \mathrm{IR}\left(\mathrm{KBr}, \mathrm{Cm}^{-1}\right): 3108,1635,1596,1522,1445$, 1399, 1341, 1318, 1292, 1232, 1211, 1181, 1144, 1052. Anal. Calcd. for $\mathrm{C}_{20} \mathrm{H}_{17} \mathrm{CIN}_{2} \mathrm{O}$ (460.92): C, 75.57; H, 3.72; N, 6.08. Found: C, 75.88; H, 3.89; N, 6.27.

1-Benzoyl-8-chloro-3-(2-naphthoyl)pyrrolo[1,2c] quinazoline (21). Yellow crystals (from $\mathrm{CHCl}, / \mathrm{EtO}) .{ }^{1} \mathrm{H}$ NMR (300 MHz, CDCl +TFA, $\delta$ ppm): 7.52-7.69 (m, 5H, Ph+ naphth), $7.72(\mathrm{dd}, 1 \mathrm{H}, J=9.0,2.1 \mathrm{~Hz}, \mathrm{H}-9), 7.76(\mathrm{~s}, 1 \mathrm{H}$, $\mathrm{H}-2), 7.88-8.00(\mathrm{~m}, 6 \mathrm{H}, \mathrm{Ph}+$ naphth), $8.12(\mathrm{~d}, 1 \mathrm{H}, J=2.1 \mathrm{~Hz}$, $\mathrm{H}-7), 8.37(\mathrm{~d}, 1 \mathrm{H}, J=1.5 \mathrm{~Hz}$, naphth), $8.85(\mathrm{~d}, 1 \mathrm{H}, J=9.0 \mathrm{~Hz}$, $\mathrm{H}-10), 10.94(\mathrm{~s}, 1 \mathrm{H}, \mathrm{H}-5) .{ }^{13} \mathrm{C}-\mathrm{NMR}(75 \mathrm{MHz}, \mathrm{CDCl}+\mathrm{TFA}, \delta$ ppm): $117.9,120.3,124.2(\mathrm{CH}), 124.7(\mathrm{CH}), 125.0,127.6$ $(\mathrm{CH}), 128.0,129.1(\mathrm{CH}), 129.2(\mathrm{CH}), 129.5(\mathrm{CH}), 130.2(\mathrm{CH})$ $131.1(\mathrm{CH}), 131.5(\mathrm{CH}), 132.3,132.7(\mathrm{CH}), 134.4,134.6$ (CH), 135.2, 135.8, 136.1, 137.5, 139.3, 142.2, 186.6 (CO), 192.9 (CO). IR $\left(\mathrm{KBr}, \mathrm{Cm}^{-1}\right): 3058,1646,1614,1524,1449$, $1399,1351,1320,1294,1209,1183,1153,1018$. Anal. Calcd. for $\mathrm{C}_{29} \mathrm{H}_{27} \mathrm{ClN}_{2} \mathrm{O}_{2}$ (460.92): C, 75.57; $\mathrm{H}, 3.72 ; \mathrm{N}, 6.08$. Found: $\mathrm{C}, 75.44 ; \mathrm{H}, 3.97 ; \mathrm{N}, 6.31$.

Ethyl 3-(4-chlorobenzoyl)-9-chloropyrrolo[1,2c] quinazoline-1-carboxylate (22). Pale yellow crystals (from CHCl ${ }_{3}$. ${ }^{1} \mathrm{H}-\mathrm{NMR}\left(300 \mathrm{MHz}, \mathrm{CDCl}_{3}+\mathrm{TFA}, \delta \mathrm{ppm}\right.$ ): 1.51 $(\mathrm{t}, 3 \mathrm{H}, J=7.1 \mathrm{~Hz}, \mathrm{Me}), 4.56\left(\mathrm{q}, 2 \mathrm{H}, J=7.1 \mathrm{~Hz}, \mathrm{CH}_{2}\right), 7.62(\mathrm{~d}$, $\left.2 \mathrm{H}, J=8.8 \mathrm{~Hz}, \mathrm{H}-3^{\prime}, \mathrm{H}-5^{\prime}\right), 7.86\left(\mathrm{~d}, 2 \mathrm{H}, J=8.8 \mathrm{~Hz}, \mathrm{H}^{\prime} 2^{\prime}, \mathrm{H}-6^{\prime}\right)$, $7.95(\mathrm{dd}, 1 \mathrm{H}, J=8.8,2.2 \mathrm{~Hz}, \mathrm{H}-7), 8.11(\mathrm{~s}, 1 \mathrm{H}, \mathrm{H}-2), 8.12$ (d, $1 \mathrm{H}, J=8.8 \mathrm{~Hz}, \mathrm{H}-8), 9.92(\mathrm{~d}, 1 \mathrm{H}, \mathrm{J}=2.2, \mathrm{H}-10) 11.07(\mathrm{~s}, 1 \mathrm{H}$, H-5). ${ }^{13} \mathrm{C}-\mathrm{NMR}(75 \mathrm{MHz}, \mathrm{CDCl}+\mathrm{TFA}, \delta \mathrm{ppm}): 14.1(\mathrm{Me})$ $63.2\left(\mathrm{CH}_{2}\right), 115.2,119.9,123.2,125.6(\mathrm{CH}), 128.2(\mathrm{CH})$, 129.3, $129.7(\mathrm{CH}), 130.8(\mathrm{CH}), 133.3(\mathrm{CH}), 134.2(\mathrm{CH})$, 134.8, 138.3, 141.2, 142.5, 162.9 (COO), 185.6 (CO). IR $\left(\mathrm{KBr}, \mathrm{Cm}^{-1}\right)$ : 3066, 1710, 1632, 1535, 1476, 1452, 1354, 1327, 1234, 1189, 1090. Anal. Calcd. for $\mathrm{C}_{2} \mathrm{H}_{2} \mathrm{Cl}_{2} \mathrm{O}_{3}$ (413.26): C, 61.03; H, 3.41; N, 6.78. Found: C, 61. 32; 3 , 3.72; N, 6.94.

Ethyl 3-(3-nitrobenzoyl)-9-chloropyrrolo [1,2c]quinazoline-1-carboxylate (23). Pale yellow crystals (from $\mathrm{CHCl}_{3}$ ). ${ }^{1} \mathrm{H}-\mathrm{NMR}(300 \mathrm{MHz}, \mathrm{CDCl}, \delta \mathrm{ppm}): 1.43(\mathrm{t}$, $3 \mathrm{H}, J=7.1 \mathrm{~Hz}, \mathrm{Me}), 4.45\left(\mathrm{q}, 2 \mathrm{H}, J=7.1 \mathrm{~Hz}, \mathrm{CH}_{2}\right), 7.75(\mathrm{dd}$, $1 \mathrm{H}, J=8.6,2.3 \mathrm{~Hz}, \mathrm{H}-8), 7.77\left(\mathrm{t}, 1 \mathrm{H}, J=7.8 \mathrm{~Hz}, \mathrm{H}^{2}-5^{\prime}\right), 7.82(\mathrm{~s}$, $1 \mathrm{H}, \mathrm{H}-2), 7.97(\mathrm{~d}, 1 \mathrm{H}, J=8.6 \mathrm{~Hz}, \mathrm{H}-7), 8.20-8.24(\mathrm{~m}, 1 \mathrm{H}, \mathrm{H}-$ $\left.4^{\prime}\right), 8.50-8.53\left(\mathrm{~m}, 1 \mathrm{H}, \mathrm{H}-6^{\prime}\right), 8.73\left(\mathrm{t}, 1 \mathrm{H}, J=1.8 \mathrm{~Hz}, \mathrm{H}-2^{\prime}\right)$, $9.84(\mathrm{~d}, 1 \mathrm{H}, J=2.3 \mathrm{~Hz}, \mathrm{H}-10), 10.31(\mathrm{~s}, 1 \mathrm{H}, \mathrm{H}-5) .{ }^{13} \mathrm{C}-\mathrm{NMR}$ (75 MHz, CDCl,$\delta \mathrm{ppm}): 14.4(\mathrm{Me}), 61.4\left(\mathrm{CH}_{2}\right), 111.9,120.8$, $122.9,124.0(\mathrm{CH}), 126.7(\mathrm{CH}), 127.3(\mathrm{CH}), 129.9(\mathrm{CH}), 130.4$ $(\mathrm{CH}), 132.1(\mathrm{CH}), 134.4,134.6(\mathrm{CH}), 136.1,138.2,139.7$, $140.2,148.3,163.2$ (COO), 182.9 (CO). IR $\left(\mathrm{KBr}, \mathrm{Cm}^{-1}\right)$ : 3061 , $1721,1639,1528,1461,1420,1346,1277,1219,1189$, 1137, 1031. Anal. Calcd. for $\mathrm{C}_{2} \mathrm{H}_{2} \mathrm{ClN}_{3} \mathrm{O}_{5}$ (423.82): C, 59.52; $H, 3.33$; N, 9.91. Found: C, 59.90; H, 3.88; N, 10.30.

Ethyl 3-(4-nitrobenzoyl)-9-chloropyrrolo[1,2c]quinazoline-1-carboxylate (24). Yellow crystals (from $\mathrm{CHCl}_{3}$ ). ${ }^{1} \mathrm{H}-\mathrm{NMR}(300 \mathrm{MHz}, \mathrm{CDCl}+\mathrm{TFA}, \delta \mathrm{ppm}): 1.50(\mathrm{t}$, $3 \mathrm{H}, J=7.1 \mathrm{~Hz}, \mathrm{Me}), 4.46\left(\mathrm{q}, 2 \mathrm{H}, \rho^{=}=7.1 \mathrm{~Hz}, \mathrm{CH}_{2}\right), 7.98(\mathrm{dd}$, $1 \mathrm{H}, J=8.8,2.1 \mathrm{~Hz}, \mathrm{H}-8), 8.07\left(\mathrm{~d}, 2 \mathrm{H}, J=8.8 \mathrm{~Hz}, \mathrm{H}-2^{\prime}, \mathrm{H}-6^{\prime}\right)$, $8.10(\mathrm{~s}, 1 \mathrm{H}, \mathrm{H}-2), 8.14(\mathrm{~d}, 1 \mathrm{H}, J=8.8 \mathrm{~Hz}, \mathrm{H}-7), 8.48(\mathrm{~d}, 2 \mathrm{H}$, $\left.J=8.8 \mathrm{~Hz}, \mathrm{H}-3^{\prime}, \mathrm{H}-5^{\prime}\right), 9.93(\mathrm{~d}, 1 \mathrm{H}, J=2.1 \mathrm{~Hz}, \mathrm{H}-10), 11.11$ (s, $1 \mathrm{H}, \mathrm{H}-5) .{ }^{13} \mathrm{C}-\mathrm{NMR}(75 \mathrm{MHz}, \mathrm{CDCl}+\mathrm{TFA}, \delta \mathrm{ppm}): 14.0$ (Me), $63.3\left(\mathrm{CH}_{2}\right), 115.3,119.9,123.4(\mathrm{CH}), 124.3(2 \mathrm{CH})$, $125.2,128.3(\mathrm{CH}), 130.0,130.3(2 \mathrm{CH}), 133.6(\mathrm{CH}), 134.4$ (CH), 134.7, 138.4, 141.9, 142,4, 150.6, 162.8 (COO), 184.5 (CO). IR $\left(\mathrm{KBr}, \mathrm{Cm}^{-1}\right)$ : 3082, 1709, 1641, 1587, 1525, 1473, $1424,1352,1269,1217,1172,1081$. Anal. Calcd. for $\mathrm{C}_{2} \mathrm{H}_{\mathrm{CIN}} \mathrm{O}_{5}$ (423.82): C, 59.52; H, 3.33; N, 9.91. Found: $\mathrm{C}$, $59.44 ; H, 3.66 ; \mathrm{N}, 10.19$.
Ethyl 9-chloro-3-(1-naphthoyl)-pyrrolo[1,2c]quinazoline-1-carboxylate (25). Pale yellow crystals (from $\mathrm{CHCl}_{3}$ Et O). ${ }^{1} \mathrm{H}-\mathrm{NMR}\left(300 \mathrm{MHz}, \mathrm{CDCl}_{3}, \delta \mathrm{ppm}\right.$ ): 1.35 $(\mathrm{t}, 3 \mathrm{H}, J=7.1 \mathrm{~Hz}, \mathrm{Me}), 4.36\left(\mathrm{q}, 2 \mathrm{H}, J=7.1 \mathrm{~Hz}, \mathrm{CH}_{2}\right), 7.55-7.59$ (m, 3H, naphth), 7.65 (s, 1H, H-2), 7.72 (dd, $1 \mathrm{H}, J=8.7,2.1$ $\mathrm{Hz}, \mathrm{H}-8), 7.76$ (dd, $1 \mathrm{H}, J=7.1,1.2 \mathrm{~Hz}$, naphth) 7.94-7.98 (m, $2 \mathrm{H}, \mathrm{H}-7+$ naphth), $8.07(\mathrm{~d}, 1 \mathrm{H} \mathrm{J}=8.2 \mathrm{~Hz}$, naphth) 8.15-8.21 (m, $1 \mathrm{H}, \mathrm{H}-\mathrm{2}^{\prime}$, naphth), $9.82(\mathrm{~d}, 1 \mathrm{H}, \mathrm{J}=2.1 \mathrm{~Hz}, \mathrm{H}-10), 10.56$ (s, $1 \mathrm{H}, \mathrm{H}-5) .{ }^{13} \mathrm{C}-\mathrm{NMR}(75 \mathrm{MHz}, \mathrm{CDCl}, \delta \mathrm{ppm}): 14.3(\mathrm{Me})$, $61.1\left(\mathrm{CH}_{2}\right), 111.5,121.0,124.3(\mathrm{CH}), 125.1,125.2(\mathrm{CH})$, $126.7(\mathrm{CH}), 127.2(\mathrm{CH}), 127.5(\mathrm{CH}), 128.5(\mathrm{CH}), 129.9(\mathrm{CH})$, $130.8,130.9,131.6(\mathrm{CH}), 131.7(\mathrm{CH}), 133.9,134.1,135.6$ (CH), $136.4(\mathrm{CH}), 138.7(\mathrm{CH}), 139.7,163.4(\mathrm{COO}), 187.1$ (CO). IR (KBr, $\left.\mathrm{Cm}^{-1}\right): 3072,1708,1625,1570,1472,1449$, $1401,1361,1327,1252,1199,1173,1088,1029$. Anal. Calcd. for $\mathrm{C}_{25} \mathrm{H}_{7} \mathrm{ClN}_{2} \mathrm{O}_{2}$ (428.88): C, 70.01; $\mathrm{H}, 4.00 ; \mathrm{N}, 6.53$. Found: C, 70.29; H, 3.89; N, 6.77.

Ethyl 9-chloro-3-(2-naphthoyl)-pyrrolo[1,2c]quinazoline-1-carboxylate (26). Pale yellow crystals (from $\left.\mathrm{CHCl}_{3} / \mathrm{Et}_{2} \mathrm{O}\right) .{ }^{1} \mathrm{H}-\mathrm{NMR}\left(300 \mathrm{MHz}, \mathrm{CDCl}_{3}, \delta \mathrm{ppm}\right):{ }^{1} \mathrm{H}-$ NMR (300 MHz, CDCl, ä ppm): $1.40\left(\mathrm{t}, 3 \mathrm{H}, J^{3^{\prime}}=7.1 \mathrm{~Hz}, \mathrm{Me}\right)$, $4.43\left(\mathrm{~g}, 2 \mathrm{H}, J=7.1 \mathrm{~Hz}, \mathrm{CH}_{2}\right), 7.59-7.69$ (m, 2H, naphth), $7.71(\mathrm{dd}, 1 \mathrm{H}, J=8.7,2.1 \mathrm{~Hz}, \mathrm{H}-8), 7.89(\mathrm{~s}, 1 \mathrm{H}, \mathrm{H}-2), 7.92-$ $8.03\left(\mathrm{~m}, 5 \mathrm{H}, \mathrm{H}-7+\right.$ naphth), 8.41 (d, $1 \mathrm{H}, J=1.1 \mathrm{~Hz}, \mathrm{H}-1^{\prime}-$ naphth), $9.84(\mathrm{~d}, 1 \mathrm{H}, J=2.1 \mathrm{~Hz}, \mathrm{H}-10), 10.32(\mathrm{~s}, 1 \mathrm{H}, \mathrm{H}-5)$. ${ }^{13} \mathrm{C}-\mathrm{NMR}(75 \mathrm{MHz}, \mathrm{CDCl}, \delta \mathrm{ppm}): 14.4(\mathrm{Me}), 61.2\left(\mathrm{CH}_{3}\right)$, $111.3,121.0,124.0,125.2(\mathrm{CH}), 127.0(\mathrm{CH}), 127.1\left(\mathrm{CH}^{2}\right)$, $127.9(\mathrm{CH}), 128.4(\mathrm{CH}), 128.6(\mathrm{CH}), 129.4(\mathrm{CH}), 129.8(\mathrm{CH})$, $130.0(\mathrm{CH}), 130.6(\mathrm{CH}), 131.6(\mathrm{CH}), 132.4,134.1,135.3$, 136.0, 138.6, $139.6(\mathrm{CH}), 163.5(\mathrm{COO}), 185.6(\mathrm{CO})$. IR ( $\mathrm{KBr}$, $\left.\mathrm{cm}^{-1}\right): 3055,1675,1626,1469,1422,1364,1320,1281$, 1227, 1184, 1107, 1004. Anal. Calcd. for $\mathrm{C}_{25} \mathrm{H}_{1} \mathrm{CIN}_{2} \mathrm{O}_{3}$ (428.88): C, 70.01; $\mathrm{H}, 4.00 ; \mathrm{N}, 6.53$. Found: $\mathrm{C}^{2}, 75.18 ;{ }^{2} \mathrm{H}^{3}$, $4.27 ; \mathrm{N}, 6.69$.

\section{Results and discussions}

The one-pot, three components microwave-assisted synthesis of a library of pyrrolo[1,2-c]quinazolines starts from various quinazolines, 2-bromoacetophenones and electron-deficient alkynes in the presence of 1,2epoxybutane which acts both as reaction medium and as acid scavenger. Halogen atoms are grafted on several starting quinazolines in order to improve specific properties of the potentially bioactive final products regarding drugtarget interactions, increasing of membrane permeability and, therefore, their bioavailability [33]

As a model for the synthesis of pyrrolo[1,2c] quinazolines, we evaluated the one-pot, three component reaction of quinazolinela, 2-bromo-2'-aceto-naphthone2d and ethyl propiolate $3 \mathrm{a}\left(\mathrm{R}^{2}=0 \mathrm{Et}\right)$ in 1,2-epoxybutane under classical heating conditions and microwave irradiation. At the end of each experiment, 1,2-epoxybutane was partly evaporated and the crystallized pyrrolo[1,2-c]quinazoline 12 was filtered off (Table 1 ).

The experimental data proved that the microwave irradiation significantly reduces the reaction time compared to the usual heating conditions and simplify the work-up of the final reaction mixture. The best conditions were reached by the microwave irradiation of reaction mixture at $120^{\circ} \mathrm{C}$ for $45 \mathrm{~min}$

We applied these conditions for the MW-assisted synthesis of a pyrrolo[1,2-c]quinazoline library starting from quinazoline and halogen-substituted quinazolines la-d, 2bromoacetophenones or 2-bromoacetonaphthones 2a-e and terminal alkynes 3a-d in 1,2-epoxybutane which plays the role of acid scavenger and reaction solvent (scheme 1 , table 2). 
Table 1

OPTIMIZATION OF REACTION CONDITIONS FOR THE SYNTHESIS OF PYRROLO[1,2-C]QUINAZOLINE 12<smiles>C#CC(=O)OCC(C)C</smiles>

\begin{tabular}{|c|c|c|c|c|}
\hline $1 \mathbf{a}$ & $2 \mathrm{~d}$ & $3 a$ & & \\
\hline Entry & Conditions ${ }^{2}$ & Temp. $\left({ }^{\circ} \mathrm{C}\right)$ & Time (min) & Yield $(\%)^{\circ}$ \\
\hline 1 & Reflux (classical heating) & 60 & 1200 & 37 \\
\hline 2 & $\mathrm{MW}$ & 100 & 30 & 28 \\
\hline 3 & MW & 100 & 60 & 37 \\
\hline 4 & MW & 120 & 30 & 39 \\
\hline 5 & MW & 120 & 45 & 42 \\
\hline
\end{tabular}

i) $2 \mathrm{mmol} \mathrm{1,2} \mathrm{mmol} \mathrm{2,2.2} \mathrm{mmol} 3 \mathrm{in} 18 \mathrm{~mL}$ 1,2-epoxybutane

b) isolated product

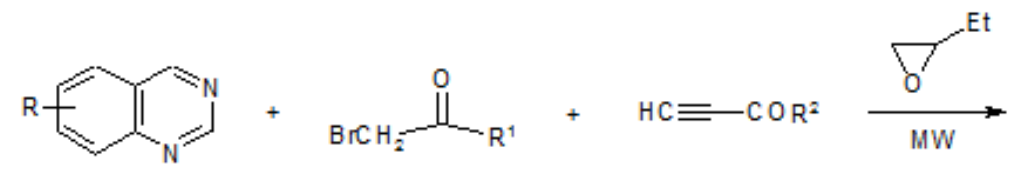

1

a: $R=H, b: R=6-F$

c. $\mathrm{R}=6-\mathrm{Cl}, \mathrm{d}: \mathrm{R}=7-\mathrm{Cl}$
3

a: $R^{2}=O E t, b: R^{2}=O M e$ c. $R^{2}=M e, d: R^{2}=P h$

$-\mathrm{CF}_{3} \mathrm{C}_{6} \mathrm{H}_{4}$ $\mathrm{R}^{1}=3,4-(\mathrm{OM} \mathrm{e})_{2} \mathrm{C}_{6} \mathrm{H}$

$R^{2}=1-$ naphthyl,
$R^{1}=2$-naphthyl,

e: $\mathrm{R}^{1}=\mathrm{Ph}$,

f: $R^{1}=3-\mathrm{NO}_{2} \mathrm{C}_{6} \mathrm{H}_{4}$

g: $\mathrm{R}^{1}=4-\mathrm{NO}_{2} \mathrm{C}_{6} \mathrm{H}_{4}$

h: $\mathrm{R}^{1}=4-\mathrm{ClC}_{6} \mathrm{H}_{4}$<smiles>[R]C=Cc1cc(C([R])=O)n2cnc3ccccc3c12</smiles>

4-26

Scheme 1. Synthesis of pyrrolo[1,2-c]quinazolines under microwave irradiation

\begin{tabular}{|c|c|c|c|c|c|}
\hline Compd. & R & $\mathbf{R}^{1}$ & $\mathrm{R}^{2}$ & $\mathrm{mp}\left({ }^{\circ} \mathrm{C}\right)$ & Yield $(\%)^{\circ}$ \\
\hline 4 & $\mathrm{H}$ & $3-\mathrm{CF}_{3} \mathrm{C}_{6} \mathrm{H}_{4}$ & OEt & $175-176$ & 44 \\
\hline 5 & $\mathrm{H}$ & $3-\mathrm{CF}_{3} \mathrm{C}_{6} \mathrm{H}_{4}$ & $\mathrm{Ph}$ & $184-186$ & 47 \\
\hline 6 & $\mathrm{H}$ & $3,4-(\mathrm{MeO})_{2} \mathrm{C}_{6} \mathrm{H}_{3}$ & OEt & $154-155$ & 42 \\
\hline 7 & $\mathrm{H}$ & $3,4-(\mathrm{MeO})_{2} \mathrm{C}_{6} \mathrm{H}_{3}$ & $\mathrm{Ph}$ & $227-229$ & 45 \\
\hline 8 & $\mathrm{H}$ & 1-naphthyl & OMe & $222-224$ & 39 \\
\hline 9 & $\mathrm{H}$ & 1-naphthyl & OEt & $227-229$ & 40 \\
\hline 10 & $\mathrm{H}$ & 1-naphthyl & $\mathrm{Me}$ & $212-214$ & 35 \\
\hline 11 & $\mathrm{H}$ & 2-naphthyl & OMe & $223-225$ & 34 \\
\hline 12 & $\mathrm{H}$ & 2-naphthyl & OEt & $199-201$ & 42 \\
\hline 13 & $\mathrm{H}$ & 2-naphthyl & $\mathrm{Ph}$ & $217-219$ & 37 \\
\hline 14 & $8-F$ & 2-naphthyl & OEt & $229-231$ & 39 \\
\hline 15 & $8-\mathrm{Cl}$ & $\mathrm{Ph}$ & $\mathrm{Ph}$ & $181-183$ & 43 \\
\hline 16 & $8-\mathrm{Cl}$ & $3-\mathrm{NO}_{2} \mathrm{C}_{6} \mathrm{H}_{4}$ & OEt & $243-245$ & 36 \\
\hline 17 & $8-\mathrm{Cl}$ & $3-\mathrm{NO}_{2} \mathrm{C}_{6} \mathrm{H}_{4}$ & $\mathrm{Ph}$ & $183-185$ & 49 \\
\hline 18 & $8-\mathrm{CI}$ & $4-\mathrm{NO}_{2} \mathrm{C}_{6} \mathrm{H}_{4}$ & $\mathrm{OEt}$ & $277-279$ & 38 \\
\hline 19 & $8-\mathrm{Cl}$ & $4-\mathrm{NO}_{2} \mathrm{C}_{6} \mathrm{H}_{4}$ & $\mathrm{Ph}$ & $257-258$ & 50 \\
\hline 20 & $8-\mathrm{Cl}$ & 1-naphthyl & $\mathrm{Ph}$ & $204-206$ & 46 \\
\hline 21 & $8-\mathrm{Cl}$ & 2-naphthyl & $\mathrm{Ph}$ & $191-193$ & 47 \\
\hline 22 & $9-\mathrm{Cl}$ & $4-\mathrm{ClC}_{6} \mathrm{H}_{4}$ & OEt & $222-224$ & 35 \\
\hline 23 & $9-\mathrm{Cl}$ & $3-\mathrm{NO}_{2} \mathrm{C}_{6} \mathrm{H}_{4}$ & OEt & $233-235$ & 32 \\
\hline 24 & 9-Cl & $4-\mathrm{NO}_{2} \mathrm{C}_{6} \mathrm{H}_{4}$ & OEt & $241-243$ & 35 \\
\hline 25 & 9-Cl & 1-naphthyl & OEt & $201-203$ & 40 \\
\hline 26 & 9-Cl & 2-naphthyl & OEt & $188-190$ & 41 \\
\hline
\end{tabular}

Table 2

NEW SYNTHESIZED PYRROLO[1,2-c]QUINAZOLINES
The reaction pathway implies the in situ formation of the quinazolinium salt, the in situ generation of the corresponding quinazolinium $\mathrm{N}$-ylide from quinazolinium salt in the presence of 1,2-epoxybutane, follow ed by the in situ1,3-dipolar cycloaddition of the quinazolinium $\mathrm{N}$-ylide with electron-deficient alkyne and dehydrogenation of the primary cycloadduct to give directly the desired pyrrolo[ $1,2-$ c] quinazoline[13,15-18].

Any substituted quinazoline can be used in this reaction, except 2-substituted quinazolines bearing large substituents because of steric hindrance of the quaternization reactions. A large range of 2-bromoacetyl derivatives or other quaternizing agents which can stabilize the intermediate $\mathrm{N}$-ylides can be used instead of 2bromoacetophenone and any available electron-deficient alkynes can be used as dipolarophiles.

The synthesized pyrrolo[1,2-c]quinazolines were structurally characterized by chemical and spectral data. The chemical shifts assignments for the protons and carbons respectively, were made based on the information obtained from bidimensional homo- and heteronuclear correlations, like COSY and HETCOR experiments. 


\section{Conclusions}

A library of pyrrolo[1,2-c]quinazoline derivatives has been successfully synthesized by combining an one-pot, three-component procedure with microwave irradiation method to easy access a library of pyrrolo[1,2c] quinazoline derivatives, starting from readily obtainable and inexpensive materials. This synthetic pathway starts from quinazolines, 2-bromoacetophenones and electrondeficient nonsymmetrical substituted alkynes in 1,2epoxybutane via 1,3-dipolar cycloaddition of quinazolinium $\mathrm{N}$-ylides.Microwave acceleration method offers valuable features such as considerable shorter reaction time, substantially reduced solvent spending, decreasing of the consumed energy and good yields. This one-pot, threecomponent microwave-assisted synthetic pathway provide a simple, clean and rapid access to a large range of pyrrolo[1,2-c]quinazoline derivatives.

\section{References}

1.BANDURCO, V.T., WONG, E.M., LEVINE, S.D., HAJOS, Z.G., J. Med. Chem., 24, 1981, p. 1455.

2.ZHENG, H.-F., YU, Z.-H., YUAN, W., TANG, Z.-L., CLOUGH, J., GU, Y.C., SHI, D.-Q., Chem. Eur. J., 20, 2014, p. 1711.

3. DUMITRASCU, F., GEORGESCU, F., GEORGESCU, E., CAIRA, M.R., Adv. Heterocycl. Chem., 129, 2019, p.155.

4. BECCALLI, E.M., MARCHESINI, A., PILATI, T., Tetrahedron, 48, 1992, p. 5359.

5. MOLINA, P., ALAJARIN, M., VIDAL, A., Tetrahedron, 51, 1995, p. 5351.

6. DORR, A.A., LUBELL, W.D., Heterocycles, 88, 2014, p. 1149.

7. PETROVICIC, M., ALESKOVIC, M., MLINARIC-MAJ ERSKI, K., Synlett, 25, 2014, p. 2769.

8. ARMSTRONG, R.J., D'ASCENZIO, M., SMITH, M.D., Synlett, 27, 2016, p. 6.

9. YANG, L.-J., CAl, H., NIE J., MA, J.-A., Eur. J. Org. Chem., 2013, p. 4434.

10. YANG, L.-J., SHEN, L., WANG, S., NIE, J., MA, J.-A., J. Org. Chem., 79, 2014, p. 3547.

11. MOLINA, P., ALLER, E., LORENZO, A., Synthesis, 1998, p. 283.

12. ZHENG, H.-F., MENG, X.-X., YUAN, W., SHI, D.-Q., Chinese J. Struct. Chem., 33, 2014, p.846.
13. GEORGESCU, E., DRUTA, I., PETROVANU, M., Rev. Roum. Chim., 26, 1981, p. 109.

14. GEORGESCU, E., GEORGESCU, F., GHEORGHIU, M., GEORGESCU, E. G., PETROVANU, M., Rev. Roum. Chim., 30, 1985, p. 611.

15. GEORGESCU, E., GEORGESCU, F., GHEORGHIU, M., FILIP, P., PETROVANU, M., Rev. Roum. Chim., 31, 1986, p. 365.

16. GEORGESCU, F., GEORGESCU, E., DRAGHICI, C., CAPROIU, M.T., Rev. Roum. Chim., 42, 1997, p. 17.

17. GEORGESCU, E., GEORGESCU, F., ROIBU, C., IUHAS, P.C., DRAGHICl, C., FILIP, P.I., Arkivoc, xii, 2008, p. 60.

18. GEORGESCU, E., GEORGESCU, F., CAIRA, M.R., NICOLESCU, A., DELEANU, C., DANILA, M.G., FILIP, P., DUMITRASCU, F., Arkivoc, xii, 2009, p. 232.

19. POPA, M.M., GEORGESCU, E., DRAGHICl, C., GEORGESCU, F., DUMITRASCU, F., DUMITRESCU, D., Monatsh. Chem., 146, 2015, p. 2029.

20. HUISGEN, R., Angew. Chem., Int. Ed., 2, 1963, p. 565.

21. ZUGRAVESCU, I., PETROVANU, M. N-Ylides Chemistry, McGrawHill Internat., New York, 1976.

22. ARMAREGO, W.L.F., J. Chem Soc., 1962, p. 561.

23. ARMAREGO, W.L.F., Adv. Heterocycl. Chem., 1, 1963, p. 253.

24. GEORGESCU, E., GEORGESCU, F., CHIRALEU, F., PETROVANU, M., Rev. Roum. Chim., 28, 1983, p. 841.

25. STRAUSS, C., TRAINOR, R., Aust. J. Chem., 48, nr. 10, 1995, p. 1665. 26. VAN DER EYCKEN, E., KAPPE, C.O., Microwave-Assisted Synthesis of Heterocycles, Springer-Verlag, Berlin, Heidelberg, 2006.

27. KAPPE, C.O., DALLINGER, D., MURPHREE, S.S.,Practical Microwave Synthesis for Organic Chemists: Strategies, Instruments, and Protocols, Wiley-VCH: Darmstadt, Germany, 2009.

28. ZBANCIOC, G., BEJAN, V., RISCA, M., MOLdOVEANU, C., MANGALAGIU, I.I., Molecules, 14, 2009, p. 403.

29. KRUITHOF, A., RUIJTER, E., ORRU, R.V.A., Curr. Org. Chem., 15, 2011, p. 204.

30. GEORGESCU, E., GEORGESCU, F., DRAGHICl, C., CRISTIAN, L., POPA, M.M., DUMITRASCU, F., Comb. Chem. High Throughput Screen.,16, 2013, p. 851.

31. KAPPE, C.O., PIEBER, B., DALLINGER, D., Angew. Chem. Int. Edit., 52, 2013, p. 1088.

32. ZBANCIOC,G., MOLDOVEANU, C.I., ZBANCIOC, A.-M., HUMELNICU, I., MANGALAGIU, I.I., Rev. Roum. Chim., 61, 2016, p. 441.

33. HERNANDES, M.Z., CAVALCANTI, S.M.T., MOREIRA,D.R.M., DE AZEVEDO, W.F. J r., LEITE, A.C.L., Curr. Drug Targets, 11, 2010, p. 303.

Manuscript received: 4.04 .2019 